\title{
Second contribution to the sawflies of Belsö Somogy (Hymenoptera: Symphyta)
}

\author{
AtTILA Haris \\ H-1076 Budapest, Garay street 19 2/20., Hungary \\ e-mail: attilaharis@yahoo.com
}

\begin{abstract}
HARIs, A.: Second contribution to the sawflies of Belsö Somogy (Hymenoptera: Symphyta).
Abstract: 105 species were collected in Belső Somogy. With the present results, the known number of sawflies of this area increased up to 164. 11 rare species were captured: Pamphilius marginatus (Serville, 1823), Dolerus (Cyperolerus) anticus ssp. anticus (Klug, 1818), Strongylogaster mixta (Klug, 1817), Strongylogaster xanthocera (Stephens, 1835), Claremontia uncta (Klug, 1816), Periclista (Periclista) albida (Klug, 1816), Periclista (Periclista) pubescens (Zaddach, 1859), Periclista (Periclista) lineolata (Klug, 1816), Rhadinoceraea (Rhadinoceraea) micans (Klug, 1816), Dineura virididorsata (Retzius, 1783) and Pristiphora (Gymnonychus) abbreviata (Hartig, 1837).
\end{abstract}

Keywords: Hymenoptera, Symphyta, fauna, Belső Somogy, Hungary.

\section{Introduction}

In 2012, we published the first investigation of the sawfly fauna of Belsö Somogy, titled: Sawflies of Belső Somogy (Hymenoptera: Symphyta) (HARIS, 2012). The first investigation resulted 1 new species for the Carpathian Basin and 1 species new for the Hungarian fauna and high number of rare species. Therefore after 5 years, we returned to this area to continue the investigation and have better picture on its sawfly fauna.

\section{Material and methods}

We spent 10 days in April and 5 days in in May and 1-1-days in July, August, September and October with collecting in 13 sampling sites in the forest area of Belsö Somogy.

In the highest collecting season, in April, the weather was extreme and 2 times broke or approached the 100 years temperature records. On 19th and 20th of April, the min-max. temperatures were $0-4$ and $0-3{ }^{\circ} \mathrm{C}$ with snowfalls. In opposite, on 1st, 2 nd and $3 \mathrm{rd}$ of April, the temperature was nearly double than the usual in this time (22-6, 23-10 and $23-8{ }^{\circ} \mathrm{C}$ ).

For identification, Zhelochovtsev's work on the sawflies of the European part of the former USSR (ZHELOCHOVTSEV 1988) was consulted. We also used some recent revisions and works to make the identifications even more precise (BLANK \& RITZAU 1998, HARIS 2006, KOCH 1988, ZOMBORI 2016). 
For the discussion of the distribution of sawflies, we consulted the book of Roller and Haris titled Sawflies of the Carpathian Basin, History and Current Research (RoLLER \& HARIS 2008), the most recent European checklist of species (TAEGER et al. 2006) augmented by other faunistic records from the Carpathian Basin (RoLler 1993, 1994, 1996, 1998, 1999a, b, c, d, e, 2000, b, c, 2001, 2004,2005, 2006a, b, ROLLER \& LuKÁš, 1999. ROLLER et al. 2006).

The higher classification of sawflies applied in this work follows the Hymenoptera part of Fauna Europaea (ACHTERBERG 20013).

The dominant collection method was the sweep netting. It was completed in May with individual collection of sawflies.

\section{Sampling sites}

1. Berzence: Alsó-Gyóta erdő (Alsó-Gyóta forest), Western side of Filagoria. Between 46¹1'45.33"N, $17^{\circ} 13^{\prime} 56.51^{\prime \prime E}$ and $46^{\circ} 11^{\prime} 49.19^{\prime \prime} \mathrm{N}, 17^{\circ} 13^{\prime} 45.97^{\prime \prime} \mathrm{E}$.

2. Bolhás: Hókamalom: Fishing pond, between $46^{\circ} 13^{\prime} 24.20^{\prime \prime} \mathrm{N}, 17^{\circ} 16^{\prime} 52.59^{\prime \prime} \mathrm{E}$ and $46^{\circ} 13^{\prime} 17.34^{\prime \prime} \mathrm{N}$, $17^{\circ} 16^{\prime} 51.48^{\prime \prime} \mathrm{E}$.

3. Iharosberény: Pine and birch forest between $46^{\circ} 20^{\prime} 56.64^{\prime \prime} \mathrm{N}, 17^{\circ} 10^{\prime} 17.83^{\prime \prime} \mathrm{E}$ and $46^{\circ} 21^{\prime} 4.87^{\prime \prime} \mathrm{N}$, $17^{\circ} 10^{\prime} 9.34^{\prime \prime} \mathrm{E}$.

4. Kaszó: Kanizsaberek North, between $46^{\circ} 19^{\prime} 42.33^{\prime \prime N}, 17^{\circ} 11^{\prime} 54.91$ "E and $46^{\circ} 19^{\prime} 46.80^{\prime \prime N}, 17^{\circ} 12^{\prime} 6.18^{\prime \prime E}$.

5. Kaszó: Pine forest between $46^{\circ} 20^{\prime} 25.47^{\prime \prime} \mathrm{N}, 17^{\circ} 12^{\prime} 0.59^{\prime \prime} \mathrm{E}$ and $46^{\circ} 20^{\prime} 20.33^{\prime \prime N}, 1^{\circ} 11^{\prime} 58.39^{\prime \prime} \mathrm{E}$.

6. Nagyatád: Somogyszob Street lake, between $46^{\circ} 14^{\prime} 9.69^{\prime \prime} \mathrm{N}, 17^{\circ} 21^{\prime} 1.58^{\prime \prime} \mathrm{E}$ and $46^{\circ} 14^{\prime} 16.34^{\prime \prime} \mathrm{N}$, $17^{\circ} 21^{\prime} 7.14 " \mathrm{E}$

7. Segesd: Pine line $46^{\circ} 19^{\prime} 50.12^{\prime \prime N}, 17^{\circ} 20^{\prime} 6.27^{\prime \prime} \mathrm{E}$

8. Somogycsicsó: Vadetetö between $46^{\circ} 18^{\prime} 50.94^{\prime \prime N}, 17^{\circ} 10^{\prime} 29.91^{\prime \prime E}$ and 46¹8'48.04"N, 17²10'23.27"E.

9. Somogyszob: meadow at Segesd side of the village, between $46^{\circ} 17^{\prime} 58.70^{\prime \prime} \mathrm{N}, 17^{\circ} 17^{\prime} 28.07^{\prime \prime} \mathrm{E}$ and $46^{\circ} 18^{\prime} 3.10^{\prime \prime} \mathrm{N}, 17^{\circ} 17^{\prime} 29.19^{\prime \prime} \mathrm{E}$.

10. Szenta: Felső-Gyóta erdő (Felső-Gyóta forest), between $46^{\circ} 13^{\prime} 29.72^{\prime \prime N}, 17^{\circ} 15^{\prime} 5.23^{\prime \prime} \mathrm{E}$ and $46^{\circ} 13^{\prime} 7.96^{\prime \prime} \mathrm{N}$, $17^{\circ} 15^{\prime} 3.13^{\prime \prime} \mathrm{E}$

11. Szenta: Forest at Szenta-Tarany border between $46^{\circ} 13^{\prime} 5.63^{\prime \prime} \mathrm{N}, 17^{\circ} 15^{\prime} 1.05^{\prime \prime} \mathrm{E}$ and $46^{\circ} 12^{\prime} 50.62^{\prime \prime} \mathrm{N}$, $17^{\circ} 14^{\prime} 53.73^{\prime \prime} \mathrm{E}$

12. Tarany: Kettős-tó forest between $46^{\circ} 13^{\prime} 6.30^{\prime \prime} \mathrm{N}, 17^{\circ} 15^{\prime} 28.77^{\prime \prime} \mathrm{E}$ and $46^{\circ} 12^{\prime} 48.20^{\prime \prime} \mathrm{N}, 17^{\circ} 15^{\prime} 27.77^{\prime \prime} \mathrm{E}$

13. Vése, Csöpröndi út (Csöpröndi road), between $46^{\circ} 24^{\prime} 31.88^{\prime \prime} \mathrm{N}, 17^{\circ} 18^{\prime} 55.08^{\prime \prime} \mathrm{E}$ and $46^{\circ} 24^{\prime} 29.74^{\prime \prime} \mathrm{N}$, $17^{\circ} 18^{\prime} 40.02^{\prime \prime} \mathrm{E}$.

\section{Results}

\section{List of species}

\section{Pamphiliidae}

Pamphilius hortorum (Klug, 1808): Szenta: Felső-Gyóta erdő (Felső-Gyóta forest), 28. 04. 2017, 1 female. Sporadic in the Carpathian Basin, rather rare in Hungary. Larva on Rubus idaeus.

Pamphilius marginatus (Serville, 1823): Szenta: Felső-Gyóta erdő (Felső-Gyóta forest), 01. 05. 2017. Rare. Hostplants: Corylus avellana and Carpinus betulus.

Pamphilius sylvaticus (Linné, 1758): Tarany: Forest at Kettős Tó, 22. 04. 2017, 1 female; Kaszó: Kanizsaberek North, 30. 04. 2017, 1 female; Vése: Csöpröndi forest, 30. 04. 2017, 1 female. One of the commonest Pamphiliidae species. Hostplants: Sorbus aucupariae, Malus spp., Prunus spp. and Crataegus spp. 


\section{Argidae}

Aproceros leucopoda Takeuchi, 1939: Vése: Csöpröndi forest, 23. 04. 2017, 1 female. Frequent. Larva on Ulmus spp. Recently invaded Europe, known from Hungary, Poland, Slovakia, Austria, Romania, Ukraine and the Russian Far East.

Arge cyanocrocea (Forster, 1771): Szenta: Felső-Gyóta erdő (Felső-Gyóta forest), 14. 04. 2017, 1 male, 28. 04. 2017, 1 male, 06. 05. 2017, 1 female; Tarany: Forest at Kettős Tó, 06. 05. 2017, 1 male; Nagyatád: Lake at Somogyszob Street, 06. 05. 2017, 1 female. Common species. Known hostplants: Rubus idaeus and Sanguisorba officinalis.

Arge melanochra (Gmelin, 1790): Bolhás: Hókamalom: Fishing pond, 27. 05. 2017, 1 female. Common species. Hostplant: Crataegus oxycantha.

\section{Xiphydriidae}

Xiphydria longicollis (Geoffroy, 1785): Szenta: Felső-Gyóta erdő (Felső-Gyóta forest), 08. 10. 2017, 1 male. Sporadic. Hostplants: Acer, Quercus, Pinus and Betula spp.

\section{Cephidae}

Calameuta (Calameuta) filiformis (Eversmann, 1847): Kaszó: Kanizsaberek North, 30. 04. 2017, 1 female; Nagyatád: Lake at Somogyszob Street, 06. 05. 2017, 1 female; Vése: Csöpröndi forest, 20. 05. 2017, 1 female. Generally common species. Larva lives in stems of Arrhenaterum elatius, Phalaris arundinacea, Calamagrostis epigeios, Elytrigia repens and Phragmites communis.

Calameuta (Calameuta) pallipes (Klug, 1803): Kaszó: Kanizsaberek North, 30. 04. 2017, 1 female. Frequent species. Hostplants: diverse Poaceae.

Cephus pygmeus (Linné, 1767): Vése: Csöpröndi forest, 20. 05. 2017, 1male; Segesd: Pinus line, 27. 05. 2017, 1male. Common. Insect pest of cereals and Gramineae.

Cephus runcator (Konow, 1896): Bolhás: Hókamalom: Fishing pond, 12. 05. 2017, 1 female; Vése: Csöpröndi forest, 12. 05. 2017, 1 female; Somogyszob: meadow at Segesd side of the village, 27. 05. 2017, 1 female; Nagyatád: Lake at Somogyszob Street, 27. 05. 2017, 1 female. Sporadic.

\section{Tenthredinidae}

\section{Dolerinae}

Dolerus (Cyperolerus) anticus ssp. anticus (Klug, 1818): Vése: Csöpröndi forest, 23. 04. 2017, 1 female. Rare. Larva on Eleocharis palustris.

Dolerus (Dolerus) bajulus (Serville, 1823) (Dolerus aericeps Thomson, 1871): Bolhás: Hókamalom: Fishing pond, 12. 06. 2017, 1 female. Frequent. Larva on Equisetum palutris.

Dolerus (Achaetoprion) madidus (Klug, 1818): Szenta: Felső-Gyóta erdő (FelsőGyóta forest), 08. 04. 2017, 1 female, 1 male; Sporadic. Larva on Juncus effusus.

Dolerus (Achaetoprion) triplicatus (Klug, 1818): Szenta: Felső-Gyóta erdő (FelsőGyóta forest), 01. 04. 2017, 1 male, 08. 04. 2017, 2 females, 1 male, 22. 04. 2017, 1 female, 06. 05. 2017, 1 female Tarany: Forest at Kettős Tó, 22. 04. 2017, 1 female. Sporadic, locally frequent. Larva on Juncus filiformis and J. effusus.

Dolerus (Dicrodolerus) vestigialis (Klug, 1818): Kaszó: Kanizsaberek North, 23. 04. 2017, 1male; Szenta: Felső-Gyóta erdő (Felső-Gyóta forest), 01. 05. 2017, 1 female; Segesd: Pine line, 27. 05. 2017, 1 female. Common. Hostplants: Equisetum palustre, E. sylvaticum, E. arvense and E. pratense.

Dolerus (Poodolerus) aeneus (Hartig, 1837): Szenta: Felső-Gyóta erdő (Felső-Gyóta forest), 17. 04. 2017, 1 female; Vése: Csöpröndi forest, 23. 04. 2017, 1 female. Sporadic. Hostplants: Gramineae. 
Dolerus (Poodolerus) fumosus (Stephens, 1835): Somogycsicsó: Vadetető, 16. 04. 2017, 3 males; Szenta: Felső-Gyóta erdő (Felső-Gyóta forest), 17. 04. 2017, 1 male; Nagyatád: Lake at Somogyszob Street, 14. 04. 2017, 1 male; Vése: Csöpröndi forest, 23. 04. 2017, 1 male. Sporadic.

Dolerus (Poodolerus) gonager (Fabricius, 1781): Somogyszob: meadow at Segesd side of the village, 08. 04. 2017, 2 females; Szenta: Felső-Gyóta erdő (Felső-Gyóta forest), 14. 04. 2017, 2 females, 01. 05. 2017, 2 females. Common. Larva on Gramineae.

Dolerus (Poodolerus) haematodes (Schrank, 1781): Szenta: Felső-Gyóta erdő (FelsőGyóta forest), 22. 04. 2017, 1 female. Frequent. Larva on Juncus, Scirpus, Carex and Gramineae.

Dolerus (Poodolerus) niger (Linné, 1767): Tarany: Forest at Kettős Tó, 14. 04. 2017, 1 female; Szenta: Felső-Gyóta erdő (Felső-Gyóta forest), 01. 05. 2017, 1 female. Sporadic. Larva on Gramineae.

Dolerus (Poodolerus) nigratus (O. F. Müller, 1776): Szenta: Felső-Gyóta erdő (FelsőGyóta forest), 01. 04. 2017, 1 female, 08. 04. 2017, 2 females, 14. 04. 2017, 2 males, 17. 04. 2017, 1 male, 22. 04. 2017, 1 male, 01. 05. 2017, 2 females; Somogyszob: meadow at Segesd side of the village, 08. 04. 2017, 1 female, 1 male; Kaszó: Kanizsaberek North, 23. 04. 2017, 2 males; Vése: Csöpröndi forest, 23. 04. 2017, 1 male, 30. 04. 2017, 2 females. Common. Larva on Gramineae including cereals.

Dolerus (Poodolerus) picipes (Klug, 1818): Kaszó: Kanizsaberek North, 30. 04. 2017, 2 females; Bolhás: Hókamalom: Fishing pond, 06. 05. 2017, 5 females. Frequent. Larva on Gramineae.

Dolerus (Poodolerus) puncticollis (C. G. Thomson, 1871): Somogyszob: meadow at Segesd side of the village, 08. 04. 2017, 1 female; Kaszó: Pine forest, 23. 04. 2017, 1 female. Common. Larva on Gramineae including cereals.

Dolerus (Poodolerus) sanguinicollis (Klug, 1818): Szenta: Felső-Gyóta erdő (FelsőGyóta forest), 01. 05. 2017, 1 female. Sporadic.

\section{Selandrinae}

Aneugmenus coronatus (Klug, 1818): Szenta: Felsö-Gyóta erdő (Felső-Gyóta forest), 01. 05. 2017, 1 female; Tarany: Forest at Kettős Tó, 06. 05. 2017, 1 female. Sporadic. Larva on Dryopteris filix-mas, Aspidium sp., Athyrium filix-femina and Pteridium aquilinum.

Nesoselandria morio (Fabricius, 1781): Tarany: Forest at Kettős Tó, 17. 04. 2017, 1 female; Vése: Csöpröndi forest, 20. 05. 2017, 1 female; Nagyatád: Lake at Somogyszob Street, 14. 04. 2017, 1 male, 20. 05. 2017, 3 males. Frequent. Hostplants: Brachytecium reflexum, Ceratodon purpureus, Chenopodium album, Dicranum scoparium, Fragaria vesca, Hedwigia ciliata, Myosotis arvensis, Plagiomnium cuspidatum, Plagiothecium denticulatum, Polygonum aviculare, Polytrichum commune, Pseudobryum cinclidiodes, Sanionia uncinata, Stellaria media, Veronica chamaedrys and V. officinalis.

Selandria serva (Fabricius, 1793): Szenta: Felső-Gyóta erdő (Felső-Gyóta forest), 08. 04. 2017, 1male, 14. 04. 2017, 1 female, 1male; Bolhás: Hókamalom: Fishing pond, 12. 06. 2017, 1male. Frequent. Host plants: Poaceae, Carex spp. and Juncus spp.

Strongylogaster multifasciata (Geoffroy, 1985): Tarany: Forest at Kettős Tó, 22. 04. 2017, 1 female, 01. 05. 2017, 3 females, 06. 05. 2017, 3 females. Frequent. Hostplants: Dryopteris sp., Matteuccia struthiopteris, Aspidium sp., Polystichum sp. and Pteridium aquilinum.

Strongylogaster mixta (Klug, 1817): Szenta: Felső-Gyóta erdő (Felső-Gyóta forest), 14. 04. 2017, 2 males, 22. 04. 2017, 1 female, 1 male. Rare. Hostplants: Athyrium filixfemina, Pteridium aquilinum and Aspidium sp. 
Strongylogaster xanthocera (Stephens, 1835): Tarany: Forest at Kettős Tó, 17. 04. 2017, 1 female. Rare. Hostplant: Pteridium aquilinum.

\section{Allantinae}

Allantus (Emphytus) cinctus (Linné, 1758): Szenta: Felső-Gyóta erdő (Felső-Gyóta forest), 17. 04. 2017, 1 male. Frequent. Hostplants: Rosa spp.

Ametastegia (Ametastegia) equiseti (Fallén, 1808): Somogyszob: meadow at Segesd side of the village, 08. 04. 2017, 1male; Szenta: Felső-Gyóta erdő (Felső-Gyóta forest), 14. 04. 2017, 1 male, 06. 05. 2017, 1male; Vése: Csöpröndi forest, 16. 04. 2017, 1male; Tarany: Forest at Kettős Tó, 01. 05. 2017, 1 female, 12. 06. 2017, 1 male; Segesd: Pine line, 27. 05. 2017, 1male. Frequent. Larva on Chenopodium album, Lythrum salicaria, Polygonum persicaria and Rumex acetosella.

Ametastegia (Protemphytus) pallipes (Spinola, 1808): Tarany: Forest at Kettős Tó, 17. 04. 2017, 1 female. Frequent. Larva on Viola spp.

Ametastegia (Protemphytus) tener (Fallén, 1808): Szenta: Felső-Gyóta erdő (FelsőGyóta forest), 01. 04. 2017, 1 female. Frequent. Larva on Filipendula, Cirsium and Rumex spp.

Athalia bicolor (Serville, 1823): Szenta: Felső-Gyóta erdő (Felső-Gyóta forest), 28. 04. 2017, 1 female; Vése: Csöpröndi forest, 30. 04. 2017, 1 female, 20. 05. 2017, 1 female, 2 males, 27. 05. 2017, 2 females; Tarany: Forest at Kettős Tó, 06. 05. 2017, 1 male; Nagyatád: Lake at Somogyszob Street, 06. 05. 2017, 1 female, 12. 05. 2017, 1 female. Frequent. Hostplant unknown.

Athalia circularis (Klug, 1815): Tarany: Forest at Kettős Tó, 14. 04. 2017, 1 male; Bolhás: Hókamalom: Fishing pond, 14. 04. 2017, 1 female; Vése: Csöpröndi forest, 23. 04. 2017, 2 males, 20. 05. 2017,1 male, 27. 05. 2017, 1 male. Frequent. Hostplants: Arctium lappa, Ajuga reptans, Veronica beccabunga, $V$. longifolia, V. officinalis, Alliaria petiolata, Glechoma hederacea, Melampyrum, Capsella and Lycopus spp.

Athalia cordata (Serville, 1823): Somogyszob: meadow at Segesd side of the village, 08. 04. 2017, 1 female; Vése: Csöpröndi forest, 23. 04. 2017, 1 females; 3 males, 30. 04. 2017, 2 males, 12. 05. 2017, 1 male; Kaszó: Kanizsaberek North, 30. 04. 2017, 2 females; Tarany: Forest at Kettős Tó, 01. 05. 2017, 1 male, 06. 05. 2017, 1 male, 12. 06. 2017, 1 female; Szenta: Felső-Gyóta erdő (Felső-Gyóta forest), 01. 05. 2017, 1 male; Common. Larva on Misopates orontinum, Antirrhinum majus, Ajuga reptans, Teucrium scorodonia and Plantago spp.

Athalia liberta (Klug, 1815): Somogycsicsó: Vadetető, 16. 04. 2017, 1 male; Vése: Csöpröndi forest, 23. 04. 2017, 1 male. Frequent. Feeding on Alliaria petiolata, Arabidopsis thaliana, Cardamine hirsuta and Sisymbrium officinale.

Athalia rosae (Linné, 1758): Vése: Csöpröndi forest, 23. 04. 2017, 1 female, 1 male; Bolhás: Hókamalom: Fishing pond, 12. 05. 2017, 1 female; Nagyatád: Lake at Somogyszob Street, 20. 05. 2017, 2 females; Szenta: Felső-Gyóta erdő, 01. 05. 2017, 1 female. Common pest. Hostplants: Raphanus sativus, R. raphanistrum, Sinapis arvensis, Sisymbrium officinale, Armoracia rusticana, Barbarea sp., Brassica napus, B. juncea, B. rapa, B. oleracea, Tropaeolum majus, Sinapis arvensis, Alliara petiolata and Cardamine spp.

Empria liturata (Gmelin, 1790): Szenta: Felső-Gyóta erdő (Felső-Gyóta forest), 01. 04. 2017, 1 male, 14. 04. 2017, 1 female, 1 male, 17. 04. 2017, 2 males; Kaszó: Kanizsaberek North, 09. 04. 2017, 1 male, 16. 04. 2017, 1 male; Bolhás: Hókamalom: Fishing pond, 14. 04. 2017, 1 male; Vése: Csöpröndi forest, 16. 04. 2017, 1 male. Frequent. Hostplants: Fragaria and Geum spp. 
Empria parvula (Konow, 1892): Kaszó: Kanizsaberek North, 16. 04. 2017, 1 male; Tarany: Forest at Kettős Tó, 17. 04. 2017, 1 male. Sporadic.

Empria sexpunctata (Serville, 1823) (syn.: Empria klugii (Stephens, 1835)): Kaszó: Kanizsaberek North, 09. 04. 2017, 1 male, 16. 04. 2017, 1 male, 30. 04. 2017, 1 female; Bolhás: Hókamalom: Fishing pond, 14. 04. 2017, 1 male; Szenta: Felső-Gyóta erdő (FelsőGyóta forest), 17. 04. 2017, 3 males, 28. 04. 2017, 2 males, 28. 04. 2017, 1 female, 2 males, 01. 05. 2017, 1 male, 06. 05. 2017, 1 male; Tarany: Forest at Kettős Tó, 17. 04. 2017, 2 females, 1 male, 22. 04. 2017, 2 males, 29. 04. 2017, 1 female, 01. 05. 2017, 1 female; Vése: Csöpröndi forest, 16. 04. 2017, 1 female. Frequent. Larva on Geum spp.

Empria tridens (Konow, 1896): Berzence: Alsó-Gyóta erdő (Alsó-Gyóta forest): Western side of Filagoria, 01. 04.2017, 1 female; Kaszó: Kanizsaberek North, 08. 04. 2017, 1 female, 09. 04. 2017, 1 male; Tarany: Forest at Kettős Tó, 17. 04. 2017, 1 female, 1 male. Frequent. Hostplants: Geum spp. and Rubus idaeus.

Eriocampa ovata (Linné, 1761): Szenta: Felső-Gyóta erdő (Felső-Gyóta forest), 01. 05. 2017, 1 female. Frequent on Alnus glutinosa and A. incana.

Eriocampa umbratica (Klug, 1816): Tarany: Forest at Kettős Tó, 17. 04. 2017, 1 female, 22. 04. 2017, 1 female; Szenta: Felső-Gyóta erdő (Felső-Gyóta forest), 06. 05. 2017, 1 female; Bolhás: Hókamalom: Fishing pond, 06. 05. 2017, 1 female. Frequent on Alnus glutinosa and A. incana.

Monostegia abdominalis (Fabricius, 1798): Tarany: Forest at Kettős Tó, 29. 04. 2017, 1 female. Sporadic. Larva on Lysimachia spp., Anagallis spp. and Glaux maritima.

Taxonus agrorum (Fallén, 1808): Tarany: Forest at Kettős Tó, 14. 04. 2017, 1 male; Vése: Csöpröndi forest, 23. 04. 2017, 1 female. Frequent. Hostplants: Rubus idaeus and R. caesius.

\section{Heterarthrinae}

Fenusa (Kaliofenusa) ulmi (Sundevall, 1847): Tarany: Forest at Kettős Tó, 06. 05. 2017, 1 female. Rare. Hostplant: Ulmus glabra.

\section{Blennocampinae}

Claremontia alternipes (Klug, 1816): Szenta: Felső-Gyóta erdő (Felső-Gyóta forest), 01. 04. 2017, 1 female, 08. 04. 2017, 1 male, 14. 04. 2017, 1 female, 28. 04. 2017, 2 females; Tarany: Forest at Kettős Tó, 17. 04. 2017, 2 females, 22. 04. 2017, 2 females, 29. 04. 2017, 1 male, 01. 05. 2017, 1 male; Vése: Csöpröndi forest, 30. 04. 2017, 1 female; Szenta: Forest at Szenta-Tarany border, 01. 05. 2017, 1 male. Sporadic. Hostplant: Rubus idaeus.

Claremontia puncticeps (Konow, 1886): Tarany: Forest at Kettős Tó, 01. 05. 2017, 2 females. Sporadic. Hostplants: Fragaria spp., Sanguisorba spp. and Potentilla reptans.

Claremontia waldheimii (Gimmerthal, 1847): Szenta: Felső-Gyóta erdő (Felső-Gyóta forest), 14. 04. 2017, 1 male; Tarany: Forest at Kettös Tó, 29. 04. 2017, 1 female. Frequent. Hostplant: Geum urbanum.

Claremontia uncta (Klug, 1816): Tarany: Forest at Kettős Tó, 17. 04. 2017, 1 male, 22. 04. 2017, 1 male, 29. 04. 2017, 1 female, 12. 05. 2017, 1 male. Rare. Hostplant: Alchemilla spp.

Eutomostethus ephippium (Panzer, 1798): Szenta: Felső-Gyóta erdő (Felső-Gyóta forest), 14. 04. 2017, 1 female, 28. 04. 2017, 3 males, 01. 05. 2017, 2 males, 2 females, 06. 05. 2017, 5 males, 22. 04. 2017, 1 male, 20. 05.2017, 1 male; Nagyatád: Lake at Somogyszob Street, 14. 04. 2017, 1 female, 14. 04. 2017, 1 male; Kaszó: Pine forest, 16. 04. 2017, 1 female; Tarany: Forest at Kettös Tó, 17. 04. 2017, 2 females, 1 male, 29. 04. 2017, 1 female, 7 males, 12. 05. 2017, 1 female, 01. 05. 2017, 3 males, 2 females, 22. 04. 2017, 4 males, 14. 
04. 2017, 1 male, 06. 05. 2017, 7 males, 1 female, 12. 05. 2017, 7 males, 4 female; Vése: Csöpröndi forest, 30. 04. 2017, 1 female, 2 males, 23. 04. 2017, 4 males, 12. 05. 2017, 3 males, 20. 05. 2017, 1 male, 27. 05. 2017, 2 females, 1 male; Kaszó: Kanizsaberek North, 09. 04. 2017, 2 males, 30. 04. 2017, 1 male, 16. 04. 2017, 2 males; Iharosberény: Pine and birch forest, 30. 04. 2017, 1 male; Somogycsicsó: Vadetetö, 16. 04. 2017, 2 males; Somogyszob: meadow at Segesd side of the village, 08. 04. 2017, 1 male; Bolhás: Hókamalom: Fishing pond, 12. 05. 2017, 1male. Common species. Larva on Graminae.

Eutomostethus gagathinus (Klug, 1816): Szenta: Felső-Gyóta erdő (Felső-Gyóta forest), 22. 04. 2017, 1 male, 01. 05. 2017, 3 males, 06. 05. 2017, 1 male; Vése: Csöpröndi forest, 30. 04. 2017, 1 male, 20. 05. 2017, 1 male; Tarany: Forest at Kettős Tó, 12. 05. 2017, 1 female. Sporadic.

Eutomostethus luteiventris (Klug, 1816): Berzence: Alsó-Gyóta erdő (Alsó-Gyóta forest): Western side of Filagoria, 01. 04.2017, 3 females; Szenta: Felső-Gyóta erdő (FelsőGyóta forest), 01. 04. 2017, 1 female, 08. 04. 2017, 11 females, 14. 04. 2017, 18 females, 17. 04. 2017, 5 females, 22. 04. 2017, 4 females, 17. 04. 2017, 5 females, 22. 04. 2017, 4 females, 28. 04. 2017, 3 females, 01. 05. 2017, 10 females, 06. 05. 2017, 1 female; Tarany: Forest at Kettős Tó, 14. 04. 2017, 2 females, 12. 05. 2017, 1 female, 01. 05. 2017, 5 females; Vése: Csöpröndi forest, 23. 04. 2017, 1 female, 23. 04. 2017, 1 female, 30. 04. 2017, 2 females; Kaszó: Kanizsaberek North, 30. 04. 2017, 1 female; Iharosberény: Pine and birch forest, 30. 04. 2017, 1 female. Locally frequent, one of the dominant species of Belső Somogy. Larva on Juncus effusus.

Eutomostethus punctatus (Konow, 1887): Tarany: Forest at Kettős Tó, 01. 05. 2017, 8 males, 06. 05. 2017, 7 males, 12. 05. 2017, 3 females. Sporadic species, relatively frequent in Belsö Somogy. Hostplant: Carex paniculata.

Monophadnoides ruficruris (Brulle, 1832): Szenta: Felső-Gyóta erdő (Felső-Gyóta forest), 01. 04. 2017, 1 male, 17. 04. 2017, 1 female, 22. 04. 2017, 2 males, 28. 04. 2017, 1 females; Somogyszob: meadow at Segesd side of the village, 08. 04. 2017, 5 females; Vése: Csöpröndi forest, 16. 04. 2017, 1 female; Tarany: Forest at Kettős Tó, 22. 04. 2017, 1 female; Kaszó: Kanizsaberek North, 23. 04. 2017, 1 male; Kaszó: Pine forest, 23. 04. 2017, 1 male. Sporadic, but frequent in Belső Somogy. Hostplant: Rubus idaeus.

Monophadnus monticola (Hartig, 1837): Nagyatád: Lake at Somogyszob Street, 14. 04. 2017, 1 female. Frequent.Larva on Helleborus spp. and Ranunculus acris.

Monophadnus pallescens (Gmelin, 1790): Szenta: Felső-Gyóta erdő (Felső-Gyóta forest), 14. 04. 2017, 1 female; Vése: Csöpröndi forest, 23. 04. 2017, 1 female. Common. Hostplants: Ranunculus acris, $R$. repens, R. lanuginosus and Anemone nemorosa.

Periclista (Periclista) albida (Klug, 1816): Szenta: Felső-Gyóta erdő (Felső-Gyóta forest), 08. 04. 2017, 1 female. Rare. Hostplant: Quercus spp.

Periclista (Periclista) albiventris (Klug, 1816): Tarany: Forest at Kettős Tó, 29. 04. 2017, 1 female, 01. 05. 2017, 1 female. Sporadic.

Periclista (Periclista) pubescens (Zaddach, 1859): Tarany: Forest at Kettős Tó, 22. 04. 2017,1 female. Rare. Larva on Quercus spp.

Periclista (Periclista) lineolata (Klug, 1816): Vése: Csöpröndi forest, 16. 04. 2017, 1 female. Rare. Larva on Quercus spp.

Phymatocera aterrima (Klug, 1816): Szenta: Felső-Gyóta erdő (Felső-Gyóta forest), 22. 04. 2017, 1 male. Frequent. Hostplants: Polygonatum spp.

Rhadinoceraea (Rhadinoceraea) micans (Klug, 1816): Tarany: Forest at Kettős Tó, 01. 05. 2017, 1 male. Sporadic species but rare in Hungary. Reported only from Köszeg, Simontornya, Budapest and Nadap. Larva on Iris laevigatus, Iris pseudacorus and Iris spuria. 
Rhadinoceraea (Rhadinoceraea) reitteri (Konow, 1890): Szenta: Felső-Gyóta erdő (Felső-Gyóta forest), 14. 04. 2017, 1 female. Frequent. Larva on Iris pumila, I. germaica and I. sambucina.

Stethomostus fuliginosus (Schrank, 1781): Vése: Csöpröndi forest, 30. 04. 2017, 1 female; Bolhás: Hókamalom: Fishing pond, 06. 05. 2017, 1 female. Frequent. Larva on Ranunculus acris, $R$. repens and $R$. sceleratus.

\section{Tenthredininae}

Aglaostigma (Astochus) aucupariae (Klug, 1817): Somogyszob: meadow at Segesd side of the village, 08. 04. 2017, 1 female; Tarany: Forest at Kettős Tó, 14. 04. 2017, 1 male; Nagyatád: Lake at Somogyszob Street, 14. 04. 2017, 1 female; Kaszó: Kanizsaberek North, 30. 04. 2017, 1 female, Bolhás: Hókamalom: Fishing pond, 06. 05. 2017, 1 female. Common. Larva on Galium mollugo and G. boreale.

Aglaostigma (Astochus) fulvipes (Scopoli, 1763): Nagyatád: Lake at Somogyszob Street, 14. 04. 2017, 1 female, 1 male; Kaszó: Kanizsaberek North, 23. 04. 2017, 1 male, 30. 04. 2017, 1 female. Common. Larva on Galium mollugo and G. verum.

Macrophya (Macrophya) albicincta (Schrank, 1776): Kaszó: Kanizsaberek North, 30. 04. 2017, 1 female; Vése: Csöpröndi forest, 30. 04. 2017, 1 female; Szenta: Felsö-Gyóta erdő (Felső-Gyóta forest), 01. 05. 2017, 1 male. Common. Hostplants: Sambucus ebulus, S. nigra, S. racemosa, Valeriana officinalis and Viburnum opalus.

Macrophya (Macrophya) annulata (Geoffroy, 1785): Somogyszob: meadow at Segesd side of the village, 27. 05. 2017, 1 male. Frequent. Larva on Potentilla reptans, Origanum vulgare, Euphorbia, Rosa, Rubus and Sambucus spp.

Macrophya (Macrophya) duodecimpunctata (Linné, 1758): Tarany: Forest at Kettős Tó, 29. 04. 2017, 2 males, 06. 05. 2017, 1 female, 12. 05. 2017, 5 females, 5 males; Vése: Csöpröndi forest, 30. 04. 2017, 1 male; Bolhás: Hókamalom: Fishing pond, 01. 05. 2017, 1 male, 06. 05. 2017, 1 female, 27. 05. 2017, 2 females; Nagyatád: Lake at Somogyszob Street, 06. 05. 2017, 1 female; Somogyszob: meadow at Segesd side of the village, 27. 05. 2017, 1 male. Common. Hostplants: Graminae, Cyperaceae and Carex spp.

Macrophya (Macrophya) montana (Scopoli, 1763): Bolhás: Hókamalom: Fishing pond, 12. 05. 2017, 1 female, 20. 05. 2017, 2 females, 4 males, 27. 05. 2017, 1 female; Nagyatád: Lake at Somogyszob Street, 12. 05. 2017, 1 female, 20. 05. 2017, 1 male; Vése: Csöpröndi forest, 27. 05. 2017, 5 females, 1 male. Common. Hostplant: Rubus caesius.

Macrophya (Macrophya) postica (Brullé, 1832): Bolhás: Hókamalom: Fishing pond, 27. 05. 2017, 1 female. Frequent. Hostplant unknown.

Macrophya (Macrophya) rufipes (Linné, 1758): Nagyatád: Lake at Somogyszob Street, 20. 05. 2017, 1 female, 1 male. Larva on Agrimonia eupatoria and Vitis vinifera.

Macrophya (Macrophya) sanguinolenta (Gmelin, 1790): Bolhás: Hókamalom: Fishing pond, 12. 05. 2017, 1 female; 27. 05. 2017, 1 male. Frequent. Larva on Galenopsis, Senecio and Veronica.

Pachyprotasis rapae (Linné, 1767): Nagyatád: Lake at Somogyszob Street, 14. 04. 2017, 1 female; Somogycsicsó: Vadetető, 16. 04. 2017, 1 male; Szenta: Felső-Gyóta erdő (Felső-Gyóta forest), 22. 04. 2017, 1male; Kaszó: Kanizsaberek North, 23. 04. 2017, 1 male, 30. 04. 2017, 1 male; Tarany: Forest at Kettős Tó, 29. 04. 2017, 1male, 01. 05. 2017, 1 female, 06. 05. 2017, 1 male; Vése: Csöpröndi forest, 30. 04. 2017, 2 females. Common. Hostplants: Solanum tuberosum, Pedicularis palustris, Angelica sylvestris, Veronica beccabunga, Betonica officinalis, Corylus avellana, Salix caprea, Fraxinus excelsior, Tussilago farfara, Symphoricarpos albus, Scrophularia, Solidago, Verbascum, Origanum, Atropa, Sarothamnus, Senecio, Polygonum, Aspidium, Epilobium, Hypericum, Galeopsis, Mentha, Polystichum, Plantago, Quercus and Stachys spp. 
Pachyprotasis antennata (Klug, 1817): Tarany: Forest at Kettős Tó, 29. 04. 2017, 2 females. Sporadic. Larva on Filipendula ulmaria and Fraxinus spp.

Perineura rubi (Panzer, 1805): Tarany: Forest at Kettős Tó, 14. 04. 2017, 1 male. Sporadic. Hostplant unknown.

Rhogogaster scalaris (Klug, 1817): Vése: Csöpröndi forest, 20. 05. 2017, 1 female. Frequent. Hostplants: Pteridium aquilinum, Alnus glutinosa, Circaea, Prunus spp., Ranunculus spp., Rosa spp., Salix alba, S. purpurea, Stellaria spp., Filipendula ulmaria, Populus tremula, Padus spp., Betula spp., Corylus avellana and Sorbus spp.

Sciapteryx consobrina (Klug, 1816): Nagyatád: Somogyszobi Street: Lake, 01. 04. 2017, 2 females, 14. 04. 2017, 1 female; Szenta: Felső-Gyóta erdő (Felső-Gyóta forest), 14. 04. 2017, 1 female. Common. Larval hosts: Adoxa spp., Anemone spp. and Ranunculus ficaria.

Tenthredo (Endotethryx) campestris (Linné, 1758): Szenta: Felső-Gyóta erdő, 01. 05. 2017, 1 male; Bolhás: Hókamalom: Fishing pond, 01. 05. 2017, 1 male, 20. 05. 2017, 1 female. Frequent. Hostplant: Aegopodium podagraria.

Tenthredo (Tenthredella) atra (Linné, 1758): Vése: Csöpröndi forest, 30. 04. 2017, 1 female. Frequent. Larval hosts: Lamium, Mentha, Plantago, Vicia, Ranunculus, Scabiosa, Brassica and Solanum spp.

Tenthredo (Tenthredella) solitaria (Scopoli, 1763): Nagyatád: Lake at Somogyszob Street, 06. 05. 2017, 1 female, 1 male. Sporadic in Hungary. Larva on Euphorbia cyparissius.

Tenthredo (Zonuledo) distinguenda (Stein, 1885): Nagyatád: Lake at Somogyszob Street, 12. 05. 2017, 1 female. Sporadic. Hostplant unknown.

Tenthredopsis ornata (Serville, 1823) (syn. Tenthredopsis excisa (C. G. Thomson, 1870)): Kaszó: Kanizsaberek North, 30. 04. 2017, 3 female; Iharosberény: Pine and birch forest, 30. 04. 2017, 1 female. Frequent. Larva on Brachypodium sylvaticum.

Tenthredopsis nassata (Linné, 1767): Kaszó: Kanizsaberek North, 23. 04. 2017, 1 male; Szenta: Felsö-Gyóta erdő (Felsö-Gyóta forest), 06. 05. 2017, 1 male. Common. Hostplants: Dactylis glomerata, Deschampsia cespitosa, D. calmagrostis, Flexuosa spp., Holcus spp., Lolium perenne, Agropyron spp., Carex spp., Anthriscus silvestris and Artemisia spp.

Tenthredopsis stigma (Fabricius, 1798): Kaszó: Pine forest, 23. 04. 2017, 1 female; Nagyatád: Lake at Somogyszob Street, 06. 05. 2017, 2 females, 12. 05. 2017, 2 females; Szenta: Forest at Szenta-Tarany border, 20. 05. 2017, 1 female, 1 male. Frequent. Hostplant: Triticum intermedium.

\section{Nematinae}

Cladius (Priophorus) brullei (Dahlbom, 1835): Szenta: Felső-Gyóta erdő (FelsőGyóta forest), 22. 04. 2017, 1 male; Tarany: Forest at Kettős Tó, 22. 04. 2017, 3 females. Frequent. Hostplant: Rubus spp.

Cladius (Priophorus) compressicornis (Fabricius, 1804) (syn. Priophorus pallipes (Serville, 1823)): Kaszó: Kanizsaberek North, 16. 04. 2017, 1 female; Vése: Csöpröndi forest, 30. 04. 2017, 1 female. Frequent pest. Hostplants: Betula, Cotoneaster, Prunus, Rubus, Sorbus, Fragaria, Crataegus, Corylus and Rosa spp.

Cladius (Cladius) pectinicornis (Geoffroy, 1785): Tarany: Forest at Kettős Tó, 14. 04. 2017, 1 female. Common. Hostpalnts: Rosa, Fragaria, Poterium sanguisorba, Filipendula, Lamiastrum galeobdolon.

Dineura virididorsata (Retzius, 1783): Kaszó: Kanizsaberek North, 16. 04. 2017, 1 female, 23. 04. 2017, 2 females. Rare. Larva on Betula spp.

Hoplocampa crataegi (Klug, 1816): Szenta: Felső-Gyóta erdő (Felső-Gyóta forest), 28. 04. 2017, 1 female. Frequent. Hostplants: Crataegus spp. 
Mesoneura opaca (Fabricius, 1775): Szenta: Felső-Gyóta erdő (Felső-Gyóta forest), 17. 04. 2017, 1 female, 22. 04. 2017, 1 female; Tarany: Forest at Kettős Tó, 29. 04. 2017, 2 females. Sporadic. Hostplant: Quercus robur.

Nematus (Nematus) lucidus (Panzer, 1801): Kaszó: Kanizsaberek North, 16. 04. 2017, 1 male. Frequent. Larva on Crataegus spp., Prunus spinosa and ocasionally on cultivated Prunus.

Nematus (Pteronidea) myosotidis (Fabricius, 1804): Szenta: Felső-Gyóta erdő (FelsőGyóta forest), 14. 04. 2017, 1male, 17. 04. 2017, 1 male; Nagyatád: Lake at Somogyszob Street, 14. 04. 2017, 1 female. Common. Larval hosts: Onobrychis and Trifolium spp.

Nematus (Pteronidea) tibialis (Newman, 1837): Szenta: Felső-Gyóta erdő (FelsőGyóta forest), 01. 05. 2017, 1 female. Frequent. Larva on Robinia pseudacacia.

Nematus (Pteronidea) viridissimus (Möller, 1882): Tarany: Forest at Kettős Tó, 29. 04. 2017, 1 female. Sporadic. Hostplant: Alnus spp.

Pachynematus (Pachynematus) obductus (Hartig, 1837) (currently Euura obducta (Hartig, 1837)?: Vése: Csöpröndi forest, 30. 04. 2017, 1 female. Recently genus Pachynematus synonimised under Euura, therefore its valid name shall be Euura obductus Htg. or more likely Euura obducta Htg. depending on the next edition of the Symphyta Catalogue. Sporadic. Larva on Gramineae (Poa, Festuca spp.) and Carex.

Pachynematus (Pachynematus) vagus (Fabricius, 1781) (currently Euura vaga (Fabricius, 1781)?: Tarany: Forest at Kettős Tó, 14. 04. 2017, 1 female, 22. 04. 2017, 1 female; Kaszó: Kanizsaberek North, 30. 04. 2017, 1 female. Sporadic. Larva on Carex spp.

Pristiphora (Gymnonychus) abbreviata (Hartig, 1837): Kaszó: Pine forest, 16. 04. 2017, 1 female. Rare. Hostplant: Pyrus and Malus spp.

Pristiphora (Lygaeotus) pallidiventris (Fallén, 1808): Tarany: Forest at Kettős Tó, 06. 05. 2017, 1 female. Frequent. Larva on Geum, Potetilla, Rubus and Filipendula spp.

Pristiphora (Micronematus) monogyniae (Hartig, 1840): Vése: Csöpröndi forest, 23. 04. 2017, 1 female. Sporadic. Hostplant: Prunus spinosa ocasionally P. domestica.

Pristiphora (Pristiphora) armata (C. G. Thomson, 1863): Szenta: Felső-Gyóta erdő (Felső-Gyóta forest), 06. 05. 2017, 1 male. Frequent. Larva on Crataegus spp.

\section{Discussion}

747 specimen of 105 species were collected in 13 sites in the investigated area.

The following 33 species are recorded firstly from Belső Somogy: Pamphilius marginatus (Serville, 1823); Cephus runcator Konow, 1896; Dolerus (Cyperolerus) anticus ssp. anticus (Klug, 1818); Dolerus (Dolerus) bajulus Serville, 1823; Dolerus (Poodolerus) fumosus Stephens, 1835; Dolerus (Poodolerus) haematodes (Schrank, 1781); Strongylogaster mixta (Klug, 1817); Strongylogaster xanthocera (Stephens, 1835); Ametastegia (Protemphytus) pallipes (Spinola, 1808); Ametastegia (Protemphytus) tener (Fallén, 1808); Empria parvula (Konow, 1892); Monostegia abdominalis (Fabricius, 1798); Fenusa (Kaliofenusa) ulmi Sundevall, 1847; Claremontia puncticeps (Konow, 1886); Claremontia uncta (Klug, 1816); Monophadnus monticola (Hartig, 1837); Periclista (Periclista) albida (Klug, 1816); Periclista (Periclista) albiventris (Klug, 1816); Periclista (Periclista) pubescens (Zaddach, 1859); Periclista (Periclista) lineolata (Klug, 1816); Rhadinoceraea (Rhadinoceraea) reitteri Konow, 1890; Macrophya (Macrophya) rufipes (Linné, 1758); Macrophya (Macrophya) sanguinolenta (Gmelin, 1790); Pachyprotasis antennata (Klug, 1817); Rhogogaster scalaris (Klug, 1817); 
Tenthredopsis nassata (Linné, 1767); Cladius (Priophorus) brullei (Dahlbom, 1835); Cladius (Cladius) pectinicornis (Geoffroy, 1785); Dineura virididorsata (Retzius, 1783); Nematus (Nematus) lucidus (Panzer, 1801); Nematus (Pteronidea) viridissimus (Möller, 1882); Pachynematus (Pachynematus) obductus (Hartig, 1837) (Euura obducta (Hartig, 1837)) and Pristiphora (Gymnonychus) abbreviata (Hartig, 1837).

\section{Dominant species}

Eutomostethus ephippium (Panzer, 1798) with 83 collected specimens, and Eutomostethus luteiventris (Klug, 1816) with 79 collected specimens are the dominant species of the region.

This year, we collected only 4 Eriocampa umbratica (Klug, 1816) specimens due to the local gradation of its competitor, Agelastica alni (Linné, 1758).

\section{Rare species}

Pamphilius marginatus (Serville, 1823): Known in the Carpathian Basin from the following sites: Devínska Kobyla (Pozsony: Dévény), Resinár (Rasinari), Budapest, Aggtelek, Perkupa, Szin,Mecsek hgs.: Takanyó-völgy and: Růžd'ka.

Dolerus (Cyperolerus) anticus ssp. anticus (Klug, 1818): Its distribution in the Carpathian Basin: Ban. Štiavnica-Bot. Gard. (Selmecbánya), Viničky (Szöllőske), Szeged, Budapest, Kalocsa, Kecskemét, Szeged, Dunaörs, Dömsöd, Kelebia, Szabadszállás, Miskolc, Nagyvárad, Nagyfalu (Nusfalau), Ulma, Mons Alion, Arad, Subcarpathia, NE Croatia, Vukán, Pomáz, Niedzica, Lastonia. More than $60 \%$ of the specimens listed above, were collected in the 19th century.

Strongylogaster mixta (Klug, 1817): Fig. 2. From Hungary, this species is known only from Miskolc. Other places of capture from the Carpathian Basin: Javorina (Balázsvágás), Pol'ana Mt. (Polánahegy), Nagyszeben (Hermannstadt, Sibiu), Balánbányai hgs., Retyezát (Mt. Retezat), Tiszabogdány, Brebenyeszkul (Bogdan), Croatian Karst, Trzy Korony-Pieniny, Frenštát, Trojačka, Tatranská Lomnica (Tátralomnic) - Štart, Sekule (Székelyfalva), Vysoké Tatry: Zadná Javorová dolina (Magas Tátra: Hátsó Jávor völgy), Királymezö (Ust-Tschorna).

Strongylogaster xanthocera (Stephens, 1835): From Hungary, it is known only from Darány and Fenyőfő. Out of Hungary from Mošovce (Mosóc), Ihelník, Magura, Nagyszeben (Sibiu) and Bálványosfüred (Baile Bálványos).

Claremontia uncta (Klug, 1816): From Hungary, it is recorded only from Aggtelek, Dobogókő and Ócsa. In the higher mountainous part of the Carpathian Basin, it is more widely distributed: Hriňová (Herencsvölgy), Javorina (Balázsvágás), Limbach (Limpak), Mošovce (Mosóc), Štefanová (Istvánkirályfalva), Ihelník, Lúka (Vágluka), Bystrá (Szentiván)-sedlo Javorie (Jávori hágó), Rovná hol’a, Tiszabogdány, Brebenyeszkul (Bogdan), Trebusafejérpatak (Dilove), Pieniny Mts, Nový Jičín, Tatranská Lomnica (Tátralomnic) - Štart and Retyezát (Mt. Retezat).

Periclista (Periclista) albida (Klug, 1816): From Hungary, it is reported from Budapest, Aggtelek, Dobogókő and Nadap. Other places of capture in the Carpathian Basin: Lučenec (Losonc), Ihelník, Kňaží vrch (Pap kő), Buhusi, Bákó (Bacau), Klastromalja (Podmonastyr), Zakarpatskaya oblast, Hodslavice and Šašstín (Sasvár). We have also indefinite records from NE Croatia and Galicia.

Periclista (Periclista) pubescens (Zaddach, 1859): Only one specimen was collected more thand 120 years in Hungary, in Budapest. It is also rare in the Carpathian Basin: Čaradice (Csárad), Bojná (Nyitrabajna), Pata, Štúrovo (Párkány), Beluja (Belugya), Sitno (Szitnya), Kňaží vrch (Pap kő), Nagyszeben (Hermannstadt, Sibiu), Resica, Arad, Bílé Karpaty PLA: NNR Čeretoryje and Borosjenő (Ineu). 
Periclista (Periclista) lineolata (Klug, 1816): In Hungary, it is known from Simontornya, Zirc, Cekeháza and Balk. It is also known from Čifáre (Csiffár), Ihelník, Ivano-Frankivska obl., Ukrainian Carpaths, Podcetrtek and Bílé Karpaty PLA: NNR Čeretoryje, NR Hutě.

Rhadinoceraea (Rhadinoceraea) micans (Klug, 1816): Captured again in this area. We dicussed it's distribution in Haris, 2012.

Dineura virididorsata (Retzius, 1783): Fig. 3. Extremely rare in the Carpathian Basin. It is known only from Bátorligeti láp and Nagyszeben (Sibiu).

Pristiphora (Gymnonychus) abbreviata (Hartig, 1837): Although, its hostplants (Pyrus and Malus spp.) are common, this species is still rare. Known places of capture are Újszentmargita and Nagykovácsi. It was also reported from Brebenyeszkul, Ukraine.

\section{Evaluation of the sampling sites}

The investigated area is clearly separated into core and marginal areas.

The core area involves the sand-dunes consist of acidic sand. In some spots, these sand dunes are covered by clay. In deeper areas, where this clay is accumulated between dunes, it prevents the infiltration of the water to the deeper layers and supports wetlands, moors and even lakes (Fig. 4).

The sand dunes are dominantly covered with Hornbeam, Turkey oak and Sessile oak forests (Querceto robori-cerris carpinetosum and Quercetum robori-cerris pteridietosum) Ferns are the dominant underbrush of these dry areas (Fig. 5), which consists mainly Pteridium aquilinum (Bracken), Athyrium filix-femina (Lady fern) and Dryopteris filix-mas (Male Fern). In these forests birch (Fig. 6) and pine spots makes the vegetation more diverse. In the wet areas, the Alder vegetation is dominant.

In the marginal area, the sand dunes are not present, the soil is harder, generally brown earths (cambisol) holding various vegetations: oak forests (Quercus cerris and Quercus petrea) with lime trees (Tilia tomentosa), wet meadows, creek willow vegetation.

The marginal area holds the richest sawfly fauna, however, in these spots, dominantly common, ferquent and sometimes sporadic species were captured. Only 4 rare species were collected in these sites (mostly in Vése: Csöpröndi forest) of the 20 rare species.

The investigation is very important of these sampling sites to get complete picture on the sawfly fauna, however, the most valuable faunistic elements restricted to certain spots of the core area.

The richest sampling sites of the marginal area are: Bolhás: Hókamalom: Fishing pond; Somogyszob: marshy meadow at Segesd side of the village and Vése, Csöpröndi forest.

In interesting way, 70 percent of the rare faunistic elements were captured in 4 sampling sites of the investigated 31 sampling sites only (!) in 2012 and 2017, these are: Berzence: Filagoria, Szenta: Felső-Gyóta forest, Tarany: Kettős-tó forest (Fig. 1) and Kaszó: Kanizsaberek (only the small Northern part). The most valuable parts of Szenta: Felső-Gyóta forest,where 1 specimen of Blasticotoma filiceti Klug, 1834 and 1 specimen of Heptamelus ochroleucus (Stephens, 1835) were captured in 2012, was clear cut probably 1 or 2 years later (Fig. 7).

The other 21 sampling sites dominantly in the core area but also in the marginal areas resulted only few specimens of few (mostly lower than 10) species.

Usually, there is a second but definitely smaller peak of sawflies in the end of July and early August (dominantly Arge, Athalia and Tenthredo spp.). In interesting way, in this aspect of the year, we found only 2 specimens of Athalia rosae (Linné, 1758) and 2 specimens of Arge melanochra (Gmelin, 1790), only at Hókamalom: Fishing pond. 


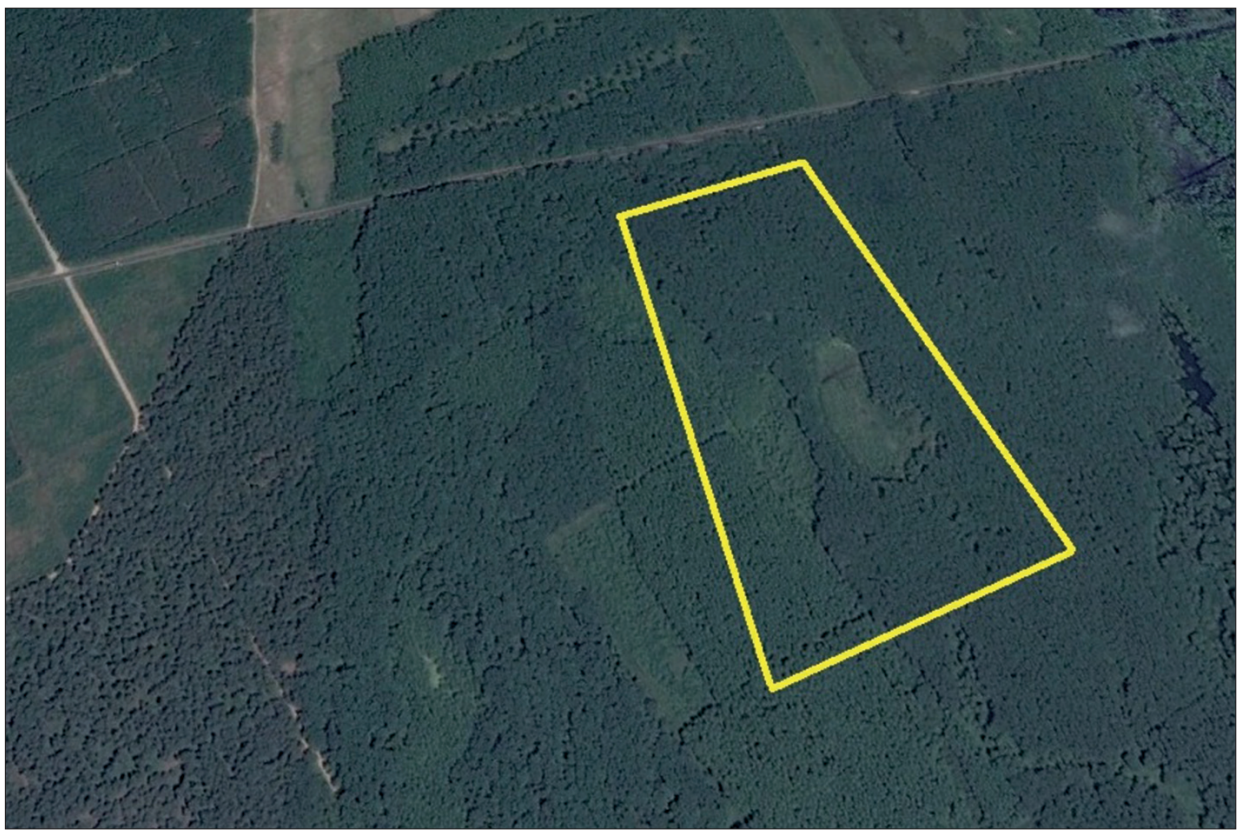

Fig. 1: Map of Tarany: Kettős Tó

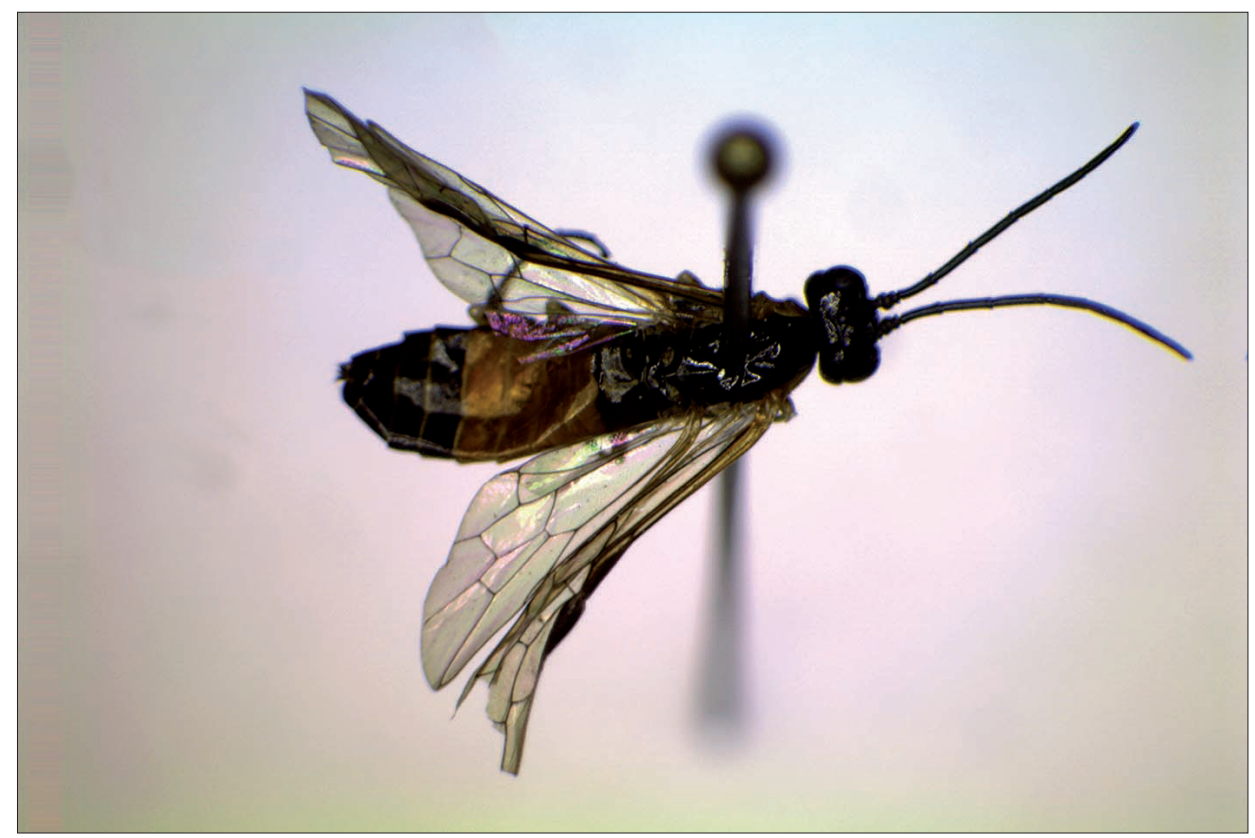

Fig. 2: Strongylogaster mixta (Klug, 1817) 


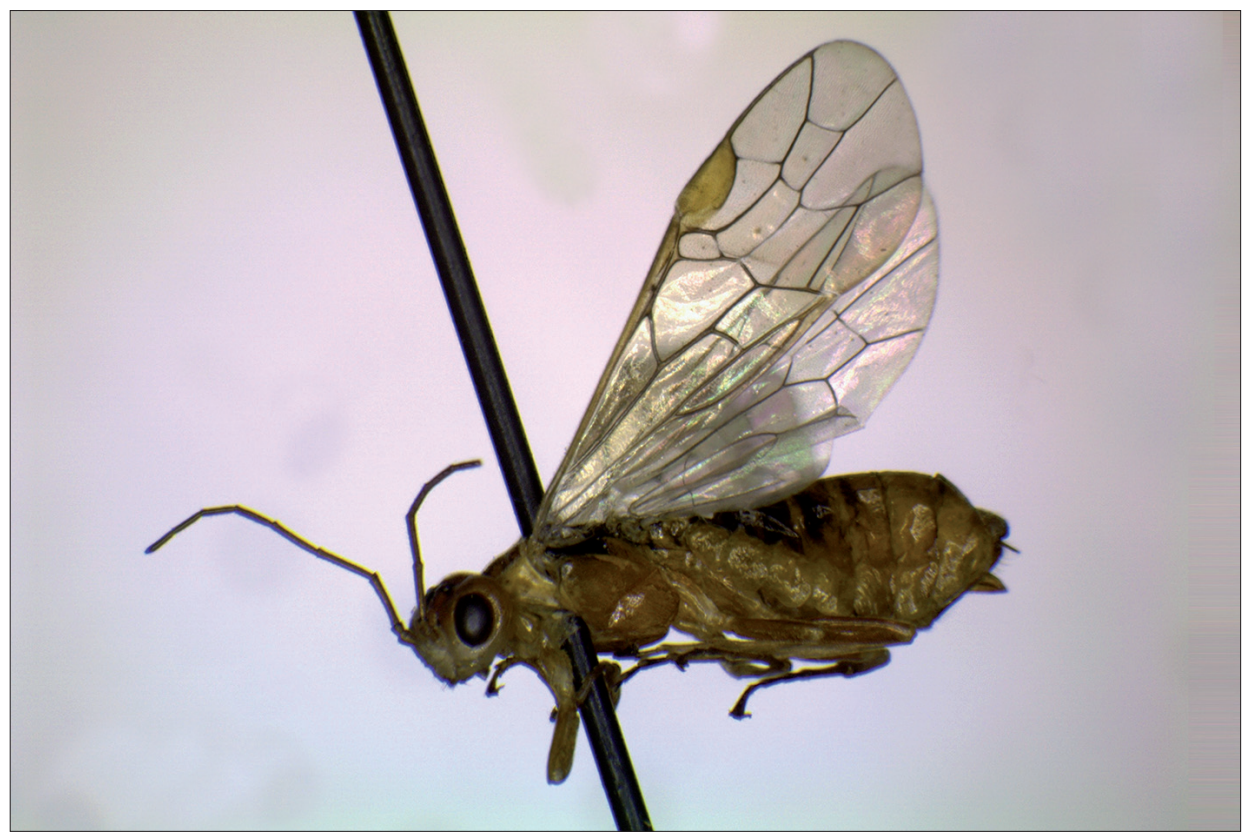

Fig. 3: Dineura virididorsata (Retzius, 1783)

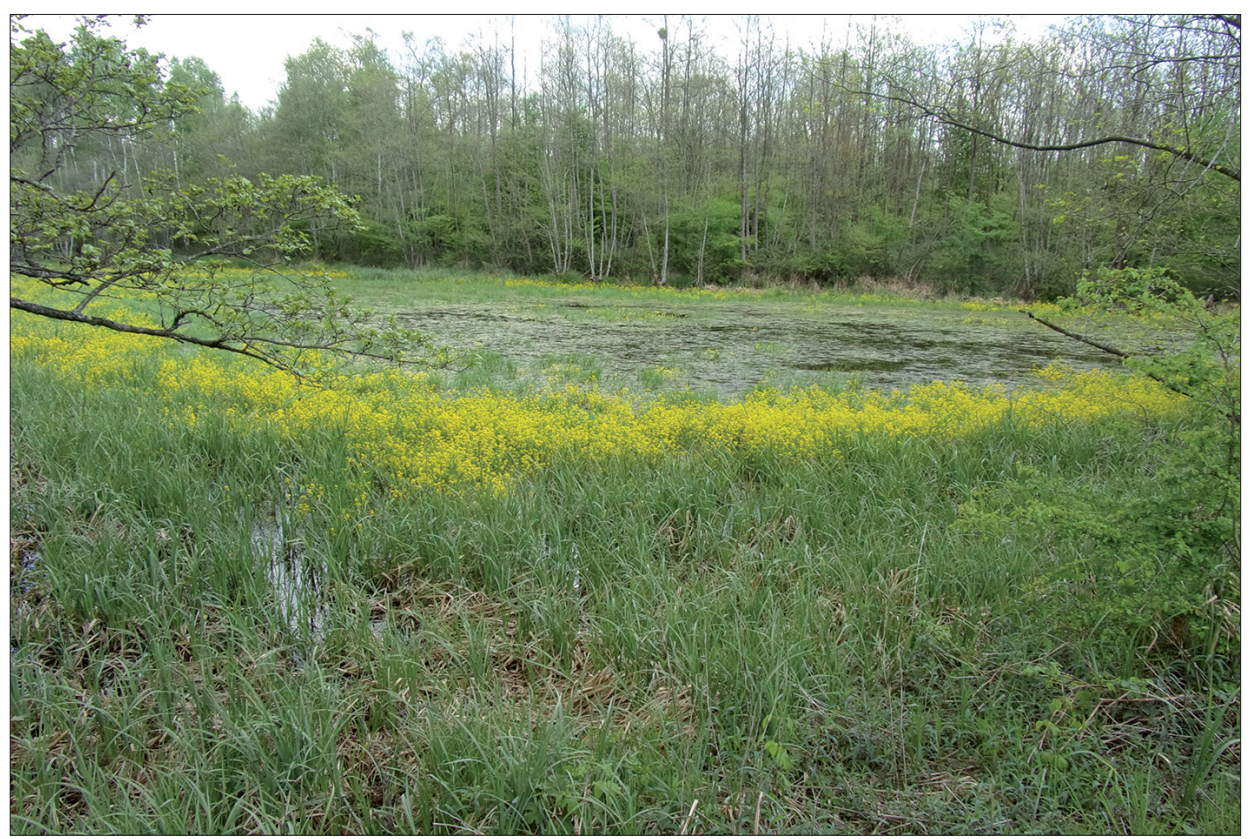

Fig. 4: Small lake on the way from Kaszó to Iharosberény 


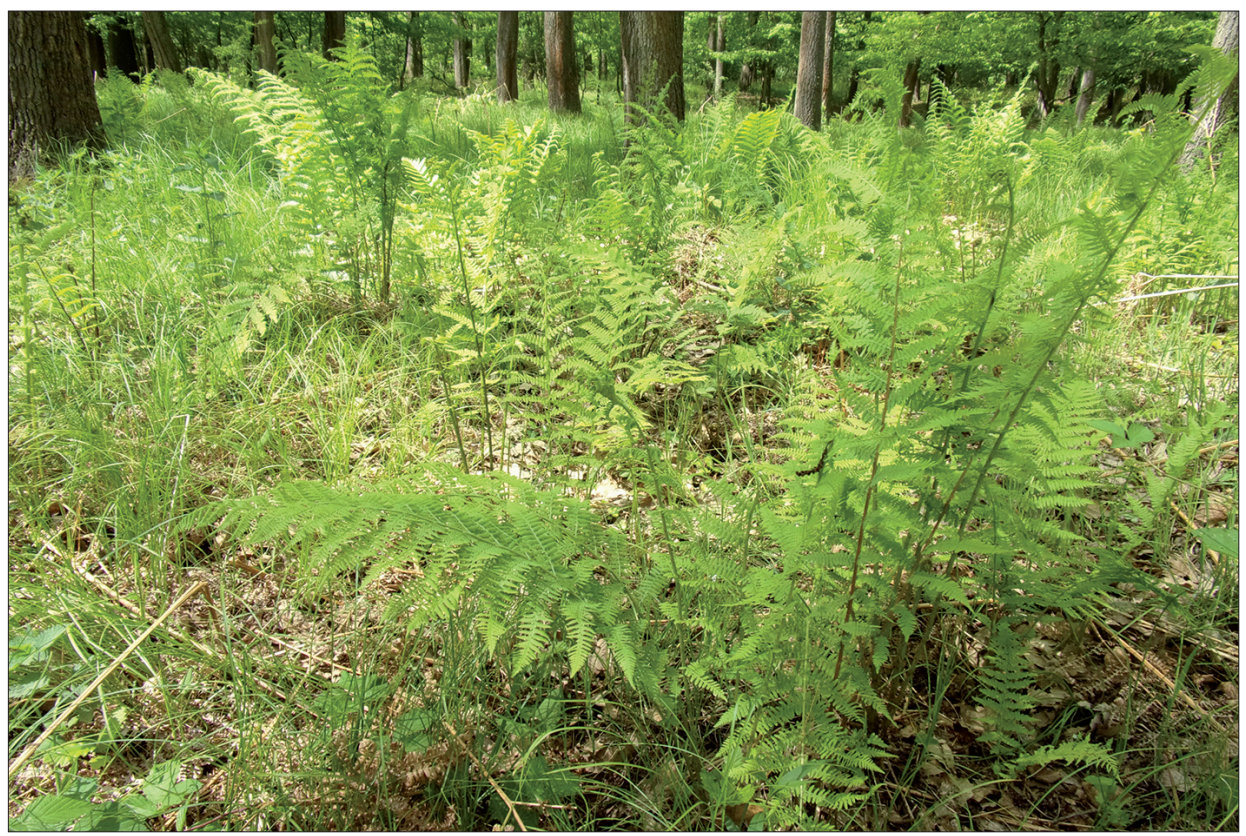

Fig. 5: Ferns are the dominant underbrushes of oak forests (Tarany: Kettős-tó)

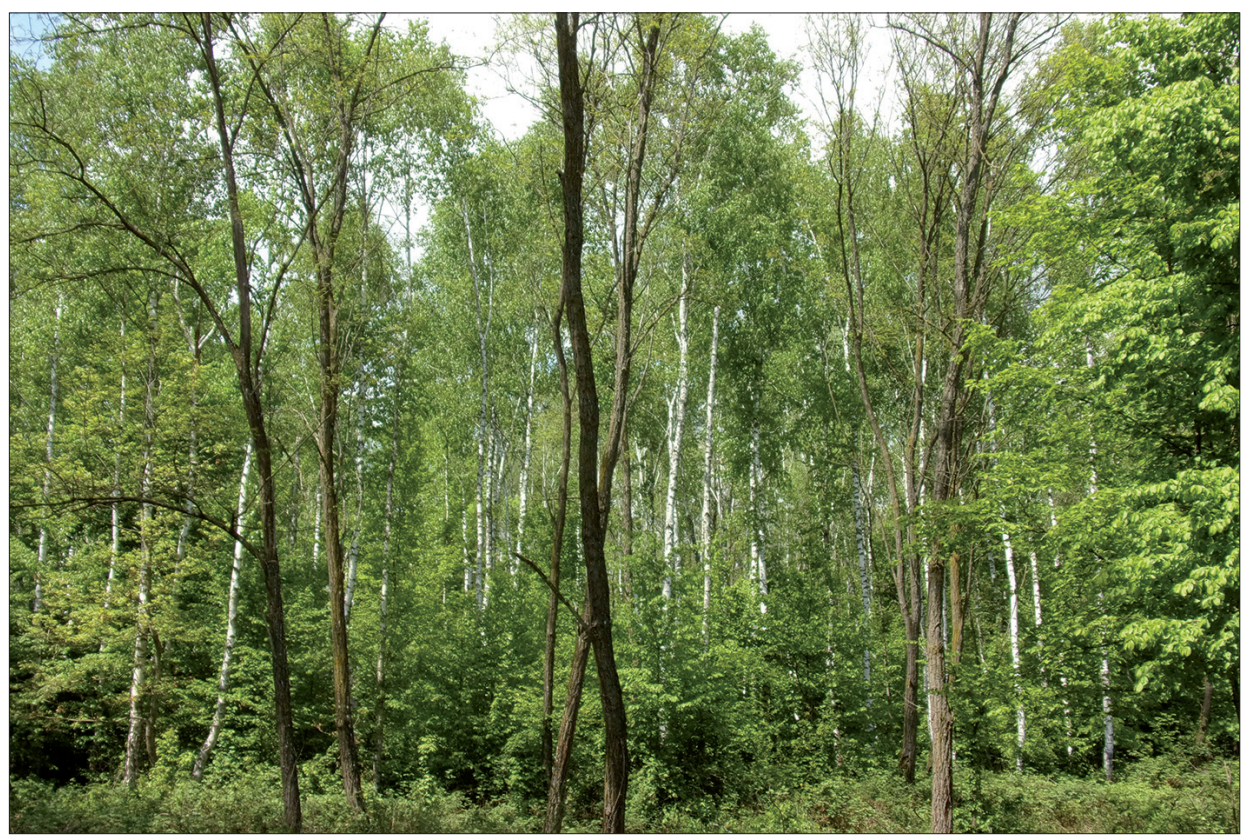

Fig. 6: Birch spot in the oak forest 


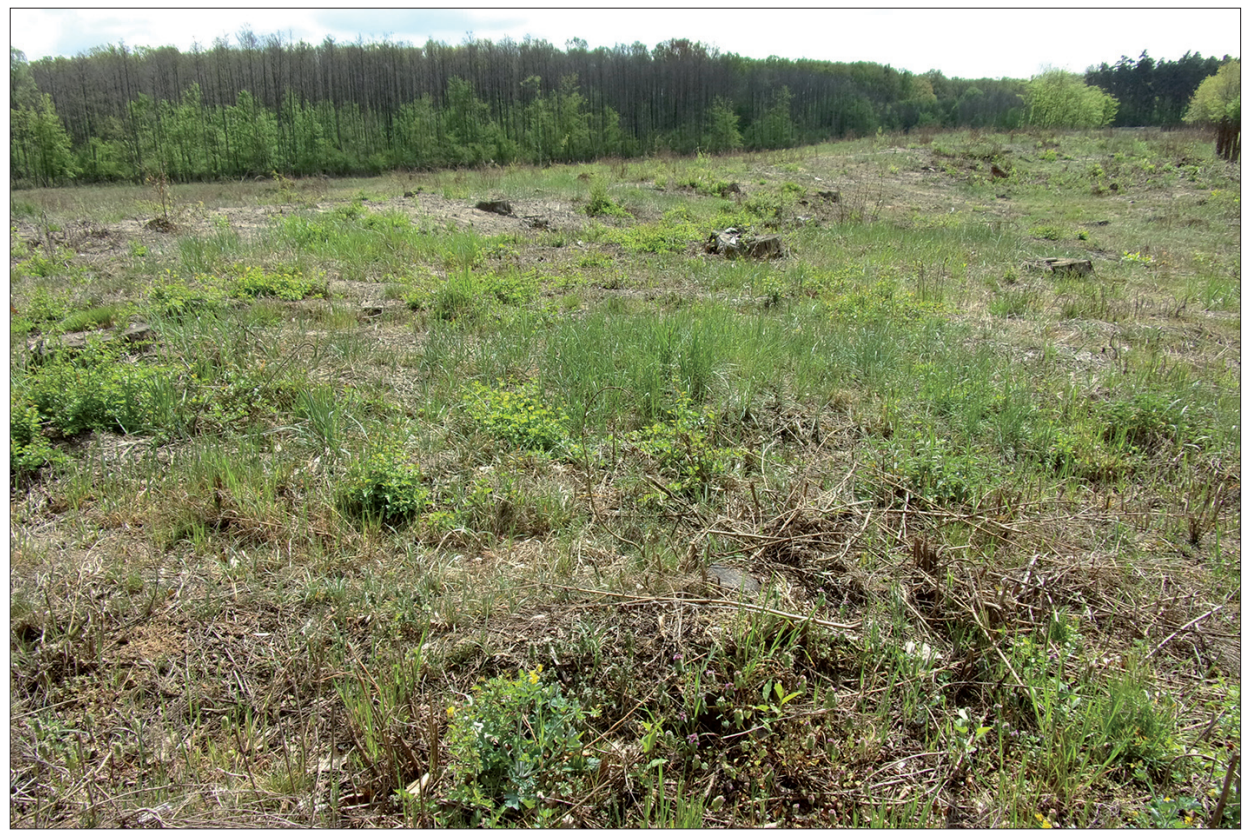

Fig 7: Clear cut at Felső-Gyóta forest 


\section{References}

ACHTERberG, C. 2013: Hymenoptera in Fauna Europaea version 2.6.2. http://www.faunaeur.org . last accessed $10^{\text {th }}$ August 2017.

Haris, A. 2012: Sawflies of Belső-Somogy (Hymenoptera: Symphyta). - Natura Somogyiensis 22:141-162.

Prous, M.; Blank, S.; Goulet, H.; Heibo, E.; Liston, A.; Malm, T.; Nyman, T.; Schmidt, S.; Smith, D.; VArdal, H.; ViitasaAri, M.; Vikberg, V. \& TAeger, A. 2014: The genera of Nematinae (Hymenoptera, Tenthredinidae) - Journal of Hymenoptera Research 40: 1-69.

Roller, L. 1993: New rec.ords of sawflies (Hymenoptera: Symphyta) from Slovakia. - Entomological Problems 24(2): 81-84.

Roller, L. 1994: Faunistics records. Symphyta. - Entomological Problems 25(2): 24.

Roller, L. 1996: New records of sawflies (Hymenoptera, Tenthredinidae) in Slovakia. - Biologia, Bratislava 51(1): 549-550.

Roller, L. 1998: Sawfly (Hymenoptera, Symphyta) community in the Devínska Kobyla National Nature Reserve. - Biologia, Bratislava 53(2): 213-221.

Roller, L. 1999a: Spoločenstvá hrubopásych (Hymenoptera: Symphyta) vybraných zoogeografických regiónov Slovenska. PhD thesis, Ústav zoológie, Slovenská akadémia vied, Bratislava, 180 pp.

Roller, L. 1999b: First records of Nematinae (Hymenoptera, Symphyta, Tenthredinidae) in Slovakia. Biologia, Bratislava 54(5): 599-600.

Roller, L. 1999c: Faunistic records from Slovakia. Hymenoptera: Symphyta: Tenthredinidae: Nematinae. Entomological Problems 30(1): 30.

Roller, L. 1999d: Faunistic records from Slovakia. Hymenoptera: Symphyta: Tenthredinidae: Nematinae. Entomological Problems 30(1): 52.

Roller, L. 1999e: Check list of the sawflies (Hymenoptera: Symphyta) of Slovakia. - Entomological Problems 30(2): 37-48.

Roller, L. 2000a Zubačkovité (Megalodontesidae) - hrubopáse xerotermov. Hmyz 1(1): 17-18.

Roller, L. 2000b First records of Blasticotomidae, Tenthredinidae, Pamphiliidae (Hymenoptera) from Slovakia. - Biologia, Bratislava 55(5): 561-562.

Roller, L. 2000c Súčasný stav poznania fauny hrubopásych (Hymenoptera, Symphyta) na Slovensku. Správy Slovenskej zoologickej spoločnosti 18: 109-114.

Roller, L. 2001: Príspevok k poznaniu hrubopásych (Hymenoptera, Symphyta) a rohačkovitých (Diptera, Sciomyzidae) prírodnej pamiatky Mitická slatina. p. 32-36. In: MásSKY, J. (ed.), Zborník výsledkov inventarizačného výskumu prírodnej pamiatky Mitická slatina. Občianske združenie Pre Prírodu, Trenčín, 99 pp.

Roller, L. 2004. Hrubopáse blanokrídlovce (Hymenoptera, Symphyta) Tematínskych kopcov. - Entomofauna Carpathica 16: 56-64.

Roller, L. 2005: Blanokrídlovce (Hymenoptera): hrubopáse (Symphyta). 117-123 In: Fauna Devínskej Kobyly. APOP, Bratislava, 181 pp.

Roller, L. 2006a: Seasonal flight activity of sawflies (Hymenoptera, Symphyta) in submontane region of the West Carpathians, Central Slovakia. - Biologia, Bratislava 61(2): 193-205.

Roller, L. 2006b: Hrubopáse blanokrídlovce (Hymenoptera, Symphyta) Tematínskych vrchov - zhrnutie faunistického výskumu. p. 53-55. In: K. RAJCOvá (ed.): Najvzácnejšie prírodné hodnoty Tematínskych vrchov. Zborník výsledkov inventarizačného výskumu územia európskeho významu Tematínske vrchy. KOZA, Trenčín a Pre Prírodu, Trenčín, 101 pp.

Roller, L. \& LukÁšs, J., 1999: New records of sawflies (Hymenoptera, Symphyta) in Slovakia. - Biologia, Bratislava 54(2): 225-228.

Roller L., Beneš K., Blank S. M., Holuša J., Jansen E., Jänicke M., Kaluza S., Kehl A., Kehr I., Kraus M., Liston A. D., Nyman T., Nie H., Savina H., Taeger A. \& Wei M., 2006: Contribution to the knowledge of sawfly fauna (Hymenoptera, Symphyta) of the Low Tatras National Park in Central Slovakia. Naturae Tutela 10: 57-72.

TAeger A. \& ViItASAARI M. 2015: European Rhogogaster s. str., with notes on several Asian species (Hymenoptera: Tenthredinidae). - Zootaxa 4013(3):369-98.

Zhelochovtsev, A. N. 1988: Otryad Hymenoptera - Pereponchatokrylye, Podotryad Symphyta Sidyachebryukhie, 7-234. In: Medvedev, K.H. (ed.) Opredelitel nasekomykh evropeiskoi chasti SSSR, Vol. 3 Hymenoptera, Part 6, Nauka, Leningrad.

Zombori, L. 2016: Levéldarázs-alkaúak IV. Tenthredinoidea IV. In Fauna Hungariae 9. Hymenoptera I. Fauna Hungariae 174. 3/c booklet. Mondat Kft. and Hungarian Natural History Museum, Budapest. 160 pp. 
